\title{
FPRG: ANOTHER VIEW AND PROPOSITIONS
}

\author{
Cédric Join , Taha Boukhobza, Frédéric Hamelin \\ and Dominique Sauter
}

\author{
Centre de Recherche en Automatique de Nancy (CRAN), \\ UMR-CNRS 7039, Université Henri Poincaré (Nancy I), \\ BP 239, 54506 Vandouvre-lès-Nancy, France. \\ E-mail: cedric.join@cran.uhp-nancy.fr
}

\begin{abstract}
This paper deals with the problem of fault detection and isolation. Existence conditions of a solution to this problem are recalled before the proposal of another solution. The proof carrying out the effectiveness of the proposed solution and illustrative examples are the main results.Copyright ${ }^{\complement} 2005$ IFAC
\end{abstract}

Keywords: Linear system, Filter, Decoupling method, FDI.

\section{INTRODUCTION}

Fault Detection and Isolation (FDI) is currently the subject of extensive researches. Two essentially stages define the FDI: residual generation and decision making. In this work, we concentrate on the residual generation aspect.

A residual generator takes the measured signals as input and gives a residual vector as output. This residual vector has to satisfy several properties which will be precisely defined in the sequel.

A systematic procedure carrying out to special observer was proposed by Beard (Beard, 1971) and Jones (Jones, 1973). Several years later a solution to FDI problem was formulated by Massoumnia (Massoumnia, 1986; Massoumnia et al., 1989) using a geometric approach.

In this paper, the main objective is to propose another form of residual generator with good properties for fault decoupling. Moreover the multifault problem can be considered by means of the directional properties of the residual since each component of the latter is only affected by one fault and not by the others ones.

In this work, the residual generator is synthesized according to input $u$, output $y$ and its derivatives which are not usually used. It is a natural extension used in nonlinear control and more precisely in input-output linearization techniques (Isidori et al., 1981; Fossard and NormandCyrot, 1995; Plestan and Glumineau, 1996; Frank and Ding, 1997; Edelmayer et al., 1999).

This paper is organized as follows. At first, the problem statement is briefly reviewed. The generators form and residual characteristics are specified. In section 3, another view of works of Massoumnia is proposed. Section 4 shows the advantage of the residual generator using derivatives of measured signals. In Section 5, an academic examples illustrate the results. Finally, in section 6 , we conclude with some suggestions for future works.

\section{PROBLEM STATEMENT}

This paper relies heavily on a few geometric concepts. The notations and terminology are identical to (Massoumnia, 1986; Massoumnia et al., 1989; Wonham, 1985) and are now quite standard. The range of $L$ is $\mathcal{L}$. By $\mathcal{S}$ and $\mathcal{S}^{\perp}$, we denote respectively the unobservable subspace and the observable subspace. $\mathcal{W}(\mathcal{L})$ denotes the set of 
all $(C, A)$-invariant subspaces containing the subspace $\mathcal{L}$, and $\mathcal{S}(\mathcal{L})$ denotes the set of all $(C, A)$ unobservability subspaces (u.o.s.) containing the subspace $\mathcal{L}$. Moreover $\mathcal{W}^{*}(\mathcal{L})$ denotes the subspace containing the infimal element of $\mathcal{W}(\mathcal{L})$. It is such that $\mathcal{W}^{*}(\mathcal{L})=\mathcal{W}^{k+1}(\mathcal{L})=\mathcal{W}^{k}(\mathcal{L})$ with:

$$
\left\{\begin{array}{l}
\mathcal{W}^{0}(\mathcal{L})=0 \\
\mathcal{W}^{k+1}(\mathcal{L})=\mathcal{L}+A\left(\mathcal{W}^{k}(\mathcal{L}) \cap \operatorname{Ker} C\right)
\end{array}\right.
$$

In other words, $\mathcal{W}^{*}(\mathcal{L})$ is the minimal $(C, A)$ invariant subspaces containing the subspace $\mathcal{L}$.

$\mathcal{S}^{*}(\mathcal{L})$ is defined by the following sequence $\mathcal{S}^{*}(\mathcal{L})=$ $\mathcal{S}^{k+1}(\mathcal{L})=\mathcal{S}^{k}(\mathcal{L})$ with:

$$
\left\{\begin{array}{l}
\mathcal{S}^{0}(\mathcal{L})=\mathcal{X} \\
\mathcal{S}^{k+1}(\mathcal{L})=\mathcal{W}^{*}(\mathcal{L})+\left(A^{-1} \mathcal{S}^{k}(\mathcal{L})\right) \cap \operatorname{Ker} C
\end{array}\right.
$$

In other words, $\mathcal{S}^{*}(\mathcal{L})$ is the minimal $(C, A)$ unobservability subspaces containing the subspace $\mathcal{L}$.

Assume that the LTI system is described by the following state-space model:

$$
\left\{\begin{array}{l}
\dot{x}=A x+B u+\sum_{i=1}^{r} L_{i} m_{i} \\
y=C x+\sum_{i=1}^{q} J_{i} n_{i}
\end{array}\right.
$$

with $x \in \mathbf{R}^{n}, u \in \mathbf{R}^{m}$ and $y \in \mathbf{R}^{p}$ are respectively state, control input and output. It is assumed that signals $u$ and $y$ are known (measured). We refer to function $m_{i}$ and $n_{i}$ which are respectively actuator and/or system faults and sensor faults. It is now admitted (Massoumnia et al., 1989) and (Park et al., 1994) that sensor faults can be represented as pseudo-actuator faults. In this paper, the model considered is the following:

$$
\left\{\begin{array}{l}
\dot{x}=A x+B u+\sum_{i=1}^{k} L_{i} m_{i} \\
y=C x
\end{array}\right.
$$

where unknown inputs $m$ represent faults (actuators, systems and sensors) and matrices $A$, $B, C$ and $L_{i}$ have been appropriately modified (compared to (3)). Using the disturbed system (4), another definition is added: $\Gamma_{y, u}$ denotes the subspace in $\mathcal{X}$ such that each element is equal $\left(\forall m_{i}\right)$ to a linear combination of $y, u$ and their derivatives. For this notion, the authors are inspired by (Kailath, 1980): p 84-90. It generalizes the observability definition formulated from nominal system. It is to be noticed that $(\operatorname{Ker} C)^{\perp} \subseteq \Gamma_{y, u} \subseteq \mathcal{S}^{\perp}$.

Remark: The system described by $A=\left(\begin{array}{cc}-1 & 0 \\ 1 & 1\end{array}\right)$, $B=L=\left(\begin{array}{ll}0 & 1\end{array}\right)^{T}$ and $C=\left(\begin{array}{ll}0 & 1\end{array}\right)$ is observable $\left(\mathcal{S}^{\perp}=\mathcal{X}\right)$ and state $x_{1}$ is not reconstructible since $\Gamma_{y, u}=\operatorname{Span}\left\{\left(\begin{array}{ll}0 & 1\end{array}\right)^{T}\right\} \neq \mathcal{X}$.
In this paper, we focus our attention on Fundamental Problem in Residual Generation (FPRG). To solve this problem, at least $k=\operatorname{dim}(m)$ (see (4)) residuals must be synthesized. Several characteristics $(5,6)$ have to be satisfied by residuals, for $i=\{1, \cdots, k\}$ :

$$
\begin{aligned}
& r_{i} \longrightarrow 0, \forall\left(u, m_{j \neq i}\right) \\
& r_{i} \neq 0, \text { with } m_{i} \neq 0
\end{aligned}
$$

The residual vector is generated according to filters. In (Massoumnia, 1986; Massoumnia et al., 1989), necessary and sufficient conditions for the solvability of the FPRG using a classical filter (7) are given.

$$
\left\{\begin{array}{l}
\dot{z}=(A+D C) z+B u-D y \\
r=H(C z-y)
\end{array}\right.
$$

where $D C z-D y$ is called the output injection. In section 3, existence conditions of a solution solving the FPRG according to residuals generated by filter (7) are recalled. Another conditions are also given and it is shown that these latter are equivalent to Massoumnia's conditions.

Based on filter form (8), another solution solving FPRG is proposed in section 4. This solution is not only no more restrictive conditions for fault isolation but also better fault decoupling capabilities.

$$
\left\{\begin{aligned}
\dot{z}= & \left(A+D\left[\begin{array}{llll}
C & C A & \cdots & C A^{(n-1)}
\end{array}\right]\right) z \\
& +B u+E\left[\begin{array}{llll}
u & u^{(1)} & \cdots & u^{(n-2)}
\end{array}\right] \\
& -D\left[\begin{array}{llll}
y & y^{(1)} & \cdots & y^{(n-1)}
\end{array}\right] \\
r= & H(z-y)
\end{aligned}\right.
$$

It is to be noticed that the output injection form is $D\left[C C A \cdots C A^{(n-1)}\right] z+E\left[\begin{array}{ll}u & u^{(1)} \\ \cdots & \left.u^{(n-2)}\right]-\end{array}\right.$ $D\left[\begin{array}{llll}y & \left.y^{(1)} \cdots y^{(n-1)}\right] & \text { where measured signals deriva- }\end{array}\right.$ tives (input and output) are used.

If $\Gamma_{y, u}$ is equal to $\mathcal{X}$ then all states $x_{i}$ (with $\forall i \in$ $\{1 \cdots n\})$ can be explained by the output injection part: $E\left[\begin{array}{lll}u & u^{(1)} \cdots u^{(n-2)}\end{array}\right]-D\left[\begin{array}{lll}y & y^{(1)} \cdots y^{(n-1)}\end{array}\right]$.

\section{ANOTHER VIEW}

Using the notations and definitions (recalled in problem statement), Theorem 3.1 gives multifault isolation conditions (see, RDDFP in (Massoumnia, 1986) or Theorem 4 in (Massoumnia et al., 1989)):

Theorem 3.1. FPRG has a solution if, and only if,

$$
\mathcal{S}^{*}\left(\overline{\mathcal{L}_{i}}\right) \cap \mathcal{L}_{i}=0, \quad i \in\{1, \cdots, k\}
$$

where $\overline{\mathcal{L}_{i}}=\sum_{j \neq i} \mathcal{L}_{i}$.

The main purpose of this section is to propose other isolation conditions without using the unobservability subspace algorithm $\left(\mathcal{S}^{*}(\cdot)\right)$. 


\subsection{Case of two faults}

For sake of simplicity, let us assume, in this section that only two faults can occur independently (i.e. $\mathrm{k}=2$ in (4)). In this case, the model is rewritten as follows:

$$
\left\{\begin{array}{l}
\dot{x}=A x+B u+L_{1} m_{1}+L_{2} m_{2} \\
y=C x
\end{array}\right.
$$

In this particular context, Theorem 3.1 implies two conditions for fault isolation:

$$
\begin{aligned}
& \mathcal{S}^{*}\left(\mathcal{L}_{2}\right) \cap \mathcal{L}_{1}=0 \\
& \mathcal{S}^{*}\left(\mathcal{L}_{1}\right) \cap \mathcal{L}_{2}=0
\end{aligned}
$$

The aim of this section is to propose equivalent conditions to the vue of relation (11), using the subspaces $\mathcal{W}(\mathcal{L})$ defined in the previous section.

(i) $\left(\mathcal{W}^{*}\left(\mathcal{L}_{2}\right)\right)^{\perp} \cap(\operatorname{Ker} C)^{\perp} \nsubseteq\left(\mathcal{W}\left(\mathcal{L}_{1}\right)\right)^{\perp}$

(ii) $\left(\mathcal{W}^{*}\left(\mathcal{L}_{1}\right)\right)^{\perp} \cap(\operatorname{Ker} C)^{\perp} \nsubseteq\left(\mathcal{W}\left(\mathcal{L}_{2}\right)\right)^{\perp}$

where $\mathcal{W}\left(\mathcal{L}_{1}\right)\left(\right.$ resp. $\left.\mathcal{W}\left(\mathcal{L}_{2}\right)\right)$ represents the $(A+$ $D C$ )-invariant subspace containing $\mathcal{L}_{1}\left(\operatorname{resp} . \mathcal{L}_{2}\right)$ and $D$ is chosen such that $\mathcal{W}^{*}\left(\mathcal{L}_{2}\right)=\inf \left(\mathcal{W}\left(\mathcal{L}_{2}\right)\right)$ $\left(\operatorname{resp} . \mathcal{W}^{*}\left(\mathcal{L}_{1}\right)=\inf \left(\mathcal{W}\left(\mathcal{L}_{1}\right)\right)\right)$.

Proposition 3.1. The following properties are equivalent:

(i) $\mathcal{S}^{*}\left(\mathcal{L}_{2}\right) \cap \mathcal{L}_{1}=0$

(ii) $\left(\mathcal{W}^{*}\left(\mathcal{L}_{2}\right)\right)^{\perp} \cap(\operatorname{Ker} C)^{\perp} \nsubseteq\left(\mathcal{W}\left(\mathcal{L}_{1}\right)\right)^{\perp}$

Proof: First, note that we have:

$$
\begin{aligned}
& \left(\mathcal{W}^{*}\left(\mathcal{L}_{2}\right)\right)^{\perp} \cap(\operatorname{Ker} C)^{\perp} \nsubseteq\left(\mathcal{W}\left(\mathcal{L}_{1}\right)\right)^{\perp} \\
& \mathcal{W}\left(\mathcal{L}_{1}\right) \nsubseteq\left(\left(\mathcal{W}^{*}\left(\mathcal{L}_{2}\right)\right)^{\perp} \cap(\operatorname{Ker} C)^{\perp}\right)^{\perp} \\
& \mathcal{W}\left(\mathcal{L}_{1}\right) \nsubseteq \mathcal{W}^{*}\left(\mathcal{L}_{2}\right)+\operatorname{Ker} C
\end{aligned}
$$

Two cases are studied:

1) $\mathcal{L}_{1} \nsubseteq \operatorname{Ker} C \Longleftrightarrow \mathcal{L}_{1} \nsubseteq \mathcal{S}$ According to $\mathcal{W}^{*}$ calculation (2), condition (13)-(i) can be replaced with: $\mathcal{W}^{*}\left(\mathcal{L}_{2}\right) \cap \mathcal{L}_{1}=$ 0 .

At the same time, $\mathcal{W}\left(\mathcal{L}_{1}\right) \nsubseteq \mathcal{W}^{*}\left(\mathcal{L}_{2}\right)+$ $\mathrm{Ker} C$ is equivalent with the following test: $\mathcal{W}\left(\mathcal{L}_{1}\right) \nsubseteq \mathcal{W}^{*}\left(\mathcal{L}_{2}\right)$.

Let us recall that $L_{1}$ is a vector, thus to consider $\mathcal{W}^{*}\left(\mathcal{L}_{2}\right) \cap \mathcal{L}_{1} \neq 0 \Longleftrightarrow \mathcal{L}_{1} \subseteq \mathcal{W}^{*}\left(\mathcal{L}_{2}\right)$ and since $\mathcal{W}^{*}\left(\mathcal{L}_{2}\right)$ is $(C, A)$-invariant, it is equivalent with $\mathcal{W}\left(\mathcal{L}_{1}\right) \subseteq \mathcal{W}^{*}\left(\mathcal{L}_{2}\right)$. Moreover $\mathcal{W}^{*}\left(\mathcal{L}_{2}\right) \cap \mathcal{L}_{1}=0 \Longleftrightarrow \mathcal{L}_{1} \nsubseteq \mathcal{W}^{*}\left(\mathcal{L}_{2}\right)$, but $\mathcal{L}_{1} \subseteq \mathcal{W}^{*}\left(\mathcal{L}_{1}\right)$ and then $\mathcal{W}\left(\mathcal{L}_{1}\right) \nsubseteq$ $\mathcal{W}^{*}\left(\mathcal{L}_{2}\right)$.

In this case the equivalence between the two propositions (13)- $(i)$ and (13)-(ii) is proven.
2) $\mathcal{L}_{1} \subseteq \operatorname{Ker} C$

Let us recall that the unobservable space is the greater $A$-invariant subspace containing $\operatorname{Ker} C\left(\operatorname{Ker} C \cap \operatorname{Ker}(C A) \cap \cdots \cap \operatorname{Ker}\left(C A^{n-1}\right)\right)$. Let us introduce $\tilde{A}=(A+D C)$ where $D$ is associated with the calculation of $\mathcal{W}^{*}\left(\mathcal{L}_{2}\right)$. Two cases are again studied.

2.1) $\tilde{A} \mathcal{L}_{1} \nsubseteq \operatorname{Ker} C \Longleftrightarrow \mathcal{L}_{1} \nsubseteq \mathcal{S}$

As previously, since $\mathcal{L}_{1}$ is included in the observable subspace, condition (13)- $(i)$ can be replaced with: $\mathcal{W}^{*}\left(\mathcal{L}_{2}\right) \cap \mathcal{L}_{1}=0$.

At the same time, $\mathcal{W}\left(\mathcal{L}_{1}\right) \nsubseteq \mathcal{W}^{*}\left(\mathcal{L}_{2}\right)+\operatorname{Ker} C$ is equivalent to following test: $\mathcal{W}\left(\mathcal{L}_{1}\right) \nsubseteq$ $\mathcal{W}^{*}\left(\mathcal{L}_{2}\right)$.

In this case, as it is previously shown, the two propositions $\mathcal{W}^{*}\left(\mathcal{L}_{2}\right) \cap \mathcal{L}_{1}=0((13)-(i))$ and $\mathcal{W}\left(\mathcal{L}_{1}\right) \nsubseteq \mathcal{W}^{*}\left(\mathcal{L}_{2}\right)((13)-(i i))$ are equivalent.

2.2) $\tilde{A} \mathcal{L}_{1} \subseteq \operatorname{Ker} C$

Two cases are studied and with a recursive reasoning on $\tilde{A}^{2} \mathcal{L}_{1}, \cdots, \tilde{A}^{n-1} \mathcal{L}_{1}$ there is still the case $\tilde{A}^{n-1} \mathcal{L}_{1} \subseteq \operatorname{Ker} C$ to study.

$2 \cdots .2) \quad \tilde{A}^{n-1} \underset{\mathcal{L}_{1}}{\mathcal{A}_{\tilde{A}}} \subseteq \operatorname{Ker} C$

Since $\tilde{A}^{\alpha} \mathcal{L}_{1} \subseteq \operatorname{Ker} C$ with $\alpha=\{1 \cdots n-$ 1) it follows that $\mathcal{L}_{1}$ is unobservable $\left(\mathcal{L}_{1} \subseteq\right.$ $\mathcal{S})$. Easily, we can conclude that $\mathcal{W}\left(\mathcal{L}_{1}\right) \subseteq$ $\operatorname{Ker} C \Longleftrightarrow \mathcal{W}\left(\mathcal{L}_{1}\right) \subseteq \mathcal{W}^{*}\left(\mathcal{L}_{2}\right)+\operatorname{Ker} C$ and $\mathcal{S}^{*}\left(\mathcal{L}_{2}\right) \cap \mathcal{L}_{1} \neq 0$.

In this case, the two propositions (13)-(i) and (13)-( $i i)$ are also equivalent.

All the cases are considered according to a recursive reasoning and (13)-(i) and (13)-(ii) are still equivalent.

Therefore, according to proposition 3.1, conditions (12) and (11) are equivalent. This constitutes another view to solve the FPRG in the case of two faults.

\subsection{Generalisation to multiple faults}

The existence conditions of a solution to FPRG proposed in the case of two faults (12) can be naturally extended to the case of multiple faults.

Theorem 3.2. FPRG has a solution if, and only if,

$$
\begin{aligned}
\left(\mathcal{W}^{*}\left(\overline{\mathcal{L}_{i}}\right)\right)^{\perp} \cap(\operatorname{Ker} C)^{\perp} & \nsubseteq\left(\mathcal{W}\left(\mathcal{L}_{i}\right)\right)^{\perp}, \\
i \in\{1, \cdots, k\} & ,
\end{aligned}
$$

where $\overline{\mathcal{L}_{i}}=\sum_{j \neq i} \mathcal{L}_{i}$.

With a proof similar to the above, the equivalence between Theorem 3.1 and Theorem 3.2 can be proved but it is not interesting to detail anymore. 


\section{PROPOSITIONS}

In this section the main objectives are:

(M1) to explain the better capability of our proposition for fault decoupling,

(M2) to prove that existence conditions of a solution solving the FPRG are no more restrictive.

These results are based on the original filter form (i.e. (8)) where the output injection is close to generalized output injection. This output injection is generally used in nonlinear feedback control (input-output linearization (Plestan and Glumineau, 1996; Glumineau et al., 1996)).

Let us introduce two new subspaces:

- the $\left(A+D\left[C C A \cdots C A^{(n-1)}\right]\right)$-invariant subspace containing $\mathcal{L}$ denoted by $\mathcal{W}_{g}(\mathcal{L})$

- $\mathcal{W}_{g}^{*}(\mathcal{L})$ : the subspace containing the infimal element of $\mathcal{W}_{g}(\mathcal{L})$ such that $\mathcal{W}_{g}^{*}(\mathcal{L})=$ $\inf \left(\mathcal{W}_{g}(\mathcal{L})\right)$ and satisfies $\mathcal{W}_{g}^{*}(\mathcal{L})=\mathcal{W}_{g}^{k+1}(\mathcal{L})=$ $\mathcal{W}_{g}^{k}(\mathcal{L})$ with:

$$
\left\{\begin{array}{l}
\mathcal{W}_{g}^{0}(\mathcal{L})=0 \\
\mathcal{W}_{g}^{k+1}(\mathcal{L})=\mathcal{L}+A\left(\mathcal{W}_{g}^{k}(\mathcal{L}) \cap \Gamma_{y, u}^{\perp}\right)
\end{array}\right.
$$

Since $\Gamma_{y, u}^{\perp} \subseteq \operatorname{Ker} C$, the following inclusions are necessarily satisfied: $\mathcal{W}_{g}^{*}(\mathcal{L}) \subseteq \mathcal{W}^{*}(\mathcal{L})$ and still $\left(\mathcal{W}^{*}(\mathcal{L})\right)^{\perp} \subseteq\left(\mathcal{W}_{g}^{*}(\mathcal{L})\right)^{\perp}$

Associated $\left(\mathcal{W}_{q}^{*}(\mathcal{L})\right)^{\perp}$ with the part of state decoupling from $L$ (fault), at least better decoupling capacities of our method are obvious. Main objective (M1) is realized.

Based on the definition of subspaces $\mathcal{W}_{g}(\cdot)$ and $\mathcal{W}_{g}^{*}(\cdot)$, the following theorem is proposed:

Theorem 4.1. FPRG has a solution if, and only if,

$$
\begin{aligned}
& \left(\mathcal{W}_{g}^{*}\left(\overline{\mathcal{L}_{i}}\right)\right)^{\perp} \cap(\operatorname{Ker} C)^{\perp} \nsubseteq\left(\mathcal{W}_{g}\left(\mathcal{L}_{i}\right)\right)^{\perp} \\
& i \in\{1, \cdots, k\}
\end{aligned}
$$

where $\overline{\mathcal{L}_{i}}=\sum_{j \neq i} \mathcal{L}_{i}$.

and the goal (M2) is reached with the proposition:

Proposition 4.1. Isolation conditions of Theorem 4.1 are no more restrictive than those proposed in Theorem 3.2.

Proof: To prove that Theorem 4.1 conditions are no more restrictive than Theorem 3.2 (or equivalently Theorem 3.1), we focus on the $k=i$ case:
$\mathcal{W}\left(\mathcal{L}_{i}\right) \nsubseteq \mathcal{W}^{*}\left(\overline{\mathcal{L}_{i}}\right)+\operatorname{Ker} C$

from Theorem 3.2

$$
\mathcal{W}_{g}\left(\mathcal{L}_{i}\right) \nsubseteq \mathcal{W}_{g}^{*}\left(\overline{\mathcal{L}_{i}}\right)+\operatorname{Ker} C
$$

from Theorem 4.1

Three cases are then studied:

1) $\mathcal{L}_{i} \subseteq \mathcal{W}_{g}^{*}\left(\overline{\mathcal{L}_{i}}\right)$

in this case, $\mathcal{L}_{i}$ is also included in $\mathcal{W}^{*}\left(\overline{\mathcal{L}_{i}}\right)$ and thus two conditions (18)-(i) and (18)-(ii) are not satisfied.

2) $\mathcal{L}_{i} \subseteq \mathcal{W}^{*}\left(\overline{\mathcal{L}_{i}}\right)$ and $\mathcal{L}_{i} \nsubseteq \mathcal{W}_{g}^{*}\left(\overline{\mathcal{L}_{i}}\right)$

in this case, condition (18)-(i) is not satisfied whereas condition (18)-(ii) can be satisfied. In this sense (18)-(ii) is at least less restrictive than the previous one.

3) $\mathcal{L}_{i} \nsubseteq \mathcal{W}^{*}\left(\overline{\mathcal{L}_{i}}\right)$

Let us denote by $D$ and $D_{g}$ matrices associated with $\mathcal{W}^{*}\left(\overline{\mathcal{L}_{i}}\right)$ and $\mathcal{W}_{g}^{*}\left(\overline{\mathcal{L}_{i}}\right)$, respectively $\tilde{A}=A+D C$ and

$\tilde{A}_{g}=\left(A+D_{g}\left[C C A \cdots C A^{(n-1)}\right]\right)$.

Let $p_{1} \cdots p_{n}$ be a basis for $\mathcal{X}$ such that:

$p_{1} \cdots p_{k 2}$ with $k 2 \in\{1 \cdots n\}$ is a basis of $\mathcal{W}^{*}\left(\overline{\mathcal{L}_{i}}\right)$

$p_{1} \cdots p_{k 1}$ with $k 1 \in\{1 \cdots k 2\}$ is a basis of $\mathcal{W}_{g}^{*}\left(\overline{\mathcal{L}_{i}}\right)$.

All matrices are then rewritten in this base. Let us now introduce the following matrices:

$$
\breve{A}=\left(\begin{array}{ll}
\breve{A}_{1} & \breve{A}_{2} \\
\breve{A}_{3} & \breve{A}_{4}
\end{array}\right)
$$

with,

$$
\begin{aligned}
& \breve{A}_{1}= \\
& \left(\begin{array}{cccccc}
\alpha_{1,1} & \cdots & \alpha_{1, k 1} & \cdots & \cdots & \alpha_{1, k 2} \\
\vdots & & \vdots & & & \vdots \\
\alpha_{k 1,1} & \cdots & \alpha_{k 1, k 1} & \cdots & \cdots & \alpha_{k 1, k 2} \\
\vdots & & \vdots & & & \vdots \\
\alpha_{k 2,1} & \cdots & \alpha_{k 2, k 1} & \cdots & \cdots & \alpha_{k 2, k 2}
\end{array}\right)
\end{aligned}
$$

and $\breve{A}_{2}$ and $\breve{A}_{4}$ are any matrix. It is to be noticed that $D$ and $D_{g}$ are synthesized such that $\tilde{A}=\breve{A}$ and $\tilde{A}=\breve{A}_{g}$ respectively with:

$$
\begin{aligned}
& \breve{A}_{3}= \\
& \left(\begin{array}{cccccc}
0 & \cdots & 0 & \beta_{1, k 1+1} & \cdots & \beta_{1, k 2} \\
\vdots & & \vdots & \vdots & & \vdots \\
0 & \cdots & 0 & \beta_{n-k 2, k 1+1} & \cdots & \beta_{n-k 2, k 2}
\end{array}\right)
\end{aligned}
$$

and all $\beta_{i, j}=0$ for $D$ whereas $\alpha_{i, j}=0$ $(k 1 \leq i \leq k 2$ and $j \leq k 1)$ for $D_{g}$.

In the case actually studied $\mathcal{L}_{i} \subseteq \operatorname{Span}\left\{p_{k 2+1}, \cdots, p_{n}\right\}$, however as previously explained, $D$ and $D_{g}$ don't affect characteristics of matrices $\breve{A}_{2}$ and $\breve{A}_{4}$ but fault propagation $\left(\mathcal{L}_{i}, \breve{A} \mathcal{L}_{i}, \cdots, \breve{A}^{n-1} \mathcal{L}_{i}\right)$ can be different for $D$ and $D_{g}$.

Two cases are then studied: 
3.1) $\mathcal{W}\left(\mathcal{L}_{i}\right) \subseteq \operatorname{Span}\left\{p_{k 2+1}, \cdots, p_{n}\right\}$ since matrix $\breve{A}_{4}$ is not changed for $D$ and $D_{g}$ then $\mathcal{W}\left(\mathcal{L}_{i}\right)=\mathcal{W}_{g}\left(\mathcal{L}_{i}\right)$. It is why, to satisfy (18)- $(i)$ is more difficult than (18)-(ii).

3.2) $\mathcal{W}\left(\mathcal{L}_{i}\right) \nsubseteq \operatorname{Span}\left\{p_{k 2+1}, \cdots, p_{n}\right\}$

Let us introduce $\mathcal{Z} . P$ the canonical projection of $\mathcal{Z}$ on $P$. According this notation, $\mathcal{W}\left(\mathcal{L}_{i}\right) \cdot\left\{p_{1}, \cdots, p_{k 2}\right\} \subseteq \mathcal{W}_{g}\left(\mathcal{L}_{i}\right) \cdot\left\{p_{1}, \cdots, p_{k 2}\right\}$. This inclusion is mainly due to the zero in $\breve{A}_{1}\left(\alpha_{i, j}=0 \quad(k 1 \leq i \leq k 2\right.$ and $j \leq k 1)$ ). The more important is that the subspace resulting of the difference between $\mathcal{W}\left(\mathcal{L}_{i}\right) \cdot\left\{p_{1}, \cdots, p_{k 2}\right\}$ and $\mathcal{W}_{g}\left(\mathcal{L}_{i}\right) \cdot\left\{p_{1}, \cdots, p_{k 2}\right\}$ is necessarily included in $\operatorname{Ker} C$ and brings no facilities for conditions (18).

For all cases, condition (18)- $(i)$ is at best equivalent with (18)-(ii) and Proposition 4.1 is thus satisfied.

\section{EXAMPLES}

To highlight the main results (M1) and (M2) of our paper, we propose to study academic exemples. Consider the system modelled by

$$
\left\{\begin{array}{l}
\dot{x}_{1}=-x_{1}+\left(u_{1}+m_{1}\right) \\
\dot{x}_{2}=x_{1}-x_{2} \\
\dot{x}_{3}=x_{1}-x_{3}+\left(u_{2}+m_{2}\right) \\
\dot{x}_{4}=x_{2}-x_{4} \\
y_{1}=x_{3} \\
y_{2}=x_{4}
\end{array}\right.
$$

$m_{1}$ and $m_{2}$ are faults to be detected and isolated. In this case, they correspond to actuator faults. We have:

$$
\operatorname{Ker} C=\operatorname{Span}\left\{\left(\begin{array}{l}
1 \\
0 \\
0 \\
0
\end{array}\right),\left(\begin{array}{l}
0 \\
1 \\
0 \\
0
\end{array}\right)\right\}
$$

and the unobservable subspace: $\mathcal{S}=\{0\}$.

\subsection{Fault decoupling}

To illustrate fault decoupling, we pose $m_{2}=0$. Using classical approach,

$$
\mathcal{W}^{*}\left(\mathcal{L}_{1}\right)=\operatorname{Span}\left\{\left(\begin{array}{l}
1 \\
0 \\
0 \\
0
\end{array}\right),\left(\begin{array}{c}
-1 \\
1 \\
1 \\
0
\end{array}\right)\right\}
$$

However, according to the following equalities,

$$
\begin{gathered}
y_{1}^{(1)}=x_{3}^{(1)}=x_{1}-x_{3}+m_{1} \\
y_{2}^{(1)}=x_{4}^{(1)}=x_{2}-x_{4} \Longrightarrow x_{2}=y_{2}^{(1)}+y_{2}
\end{gathered}
$$

$y_{2}^{(2)}=x_{4}^{(2)}=x_{1}-2 x_{2}+x_{4} \Longrightarrow x_{1}=y_{2}^{(2)}+2 y_{2}^{(1)}+y_{2}$

subspaces spanned by $\left(\begin{array}{llll}1 & 0 & 0 & 0\end{array}\right)^{\perp}$ and $\left(\begin{array}{llll}0 & 1 & 0 & 0\end{array}\right)^{\perp}$ compose $\Gamma_{y, u}=\{0\}$ and the minimal $\left(A+D\left[C C A \cdots C A^{(n-1)}\right]\right)$-invariant subspaces containing $\mathcal{L}_{i}$ (i.e. $\mathcal{W}_{g}^{*}\left(\mathcal{L}_{i}\right)$ defined by $(16)$ ) are:

$$
\mathcal{W}_{g}^{*}\left(\mathcal{L}_{1}\right)=\operatorname{Span}\left\{\left(\begin{array}{l}
1 \\
0 \\
0 \\
0
\end{array}\right)\right\}
$$

In this last case, the dimension of the state part decoupled from fault $m_{1}$ equals $\operatorname{dim}\left(\left(\mathcal{W}_{g}^{*}\left(\mathcal{L}_{1}\right)\right)^{\perp}\right)=$ 3 (see filter (31)) whereas, in the first case, equals $\operatorname{dim}\left(\left(\mathcal{W}^{*}\left(\mathcal{L}_{1}\right)\right)^{\perp}\right)=2$. The advantage of method our proposed is then obvious (see (M1) section $4)$.

\subsection{Fault isolation conditions}

Consider now, $m_{1} \neq 0$ and $m_{2} \neq 0$. Using algorithm defined by equation (2) we obtain:

$\mathcal{S}^{*}\left(\mathcal{L}_{1}\right)=\operatorname{Span}\left\{\left(\begin{array}{l}1 \\ 0 \\ 0 \\ 0\end{array}\right),\left(\begin{array}{l}0 \\ 1 \\ 1 \\ 0\end{array}\right)\right\} \mathcal{S}^{*}\left(\mathcal{L}_{2}\right)=\operatorname{Span}\left\{\left(\begin{array}{l}0 \\ 0 \\ 1 \\ 0\end{array}\right)\right\}$

Conditions of Theorem 3.1 are satisfied and faults can then be isolated.

The minimal $\left(A+D\left[C C A \cdots C A^{(n-1)}\right]\right)$ -invariant subspaces containing $\mathcal{L}_{i}$ (i.e. $\mathcal{W}_{g}^{*}\left(\mathcal{L}_{i}\right)$ defined by (16)) are:

$$
\begin{aligned}
& \mathcal{W}_{g}^{*}\left(\mathcal{L}_{1}\right)=\operatorname{Span}\left\{\left(\begin{array}{l}
1 \\
0 \\
0 \\
0
\end{array}\right)\right\} \\
& \mathcal{W}_{g}^{*}\left(\mathcal{L}_{2}\right)=\operatorname{Span}\left\{\left(\begin{array}{l}
0 \\
0 \\
1 \\
0
\end{array}\right)\right\}
\end{aligned}
$$

$\mathcal{W}_{g}\left(\mathcal{L}_{1}\right)=\operatorname{Span}\left\{\left(\begin{array}{l}1 \\ 0 \\ 0 \\ 0\end{array}\right),\left(\begin{array}{l}0 \\ 1 \\ 1 \\ 0\end{array}\right),\left(\begin{array}{l}0 \\ 0 \\ 0 \\ 1\end{array}\right)\right\}$

$$
\mathcal{W}_{g}\left(\mathcal{L}_{2}\right)=\operatorname{Span}\left\{\left(\begin{array}{l}
0 \\
0 \\
1 \\
0
\end{array}\right)\right\}
$$

and two no-inclusions are satisfied:

$$
\begin{aligned}
& \left(\mathcal{W}_{g}^{*}\left(\mathcal{L}_{1}\right)\right)^{\perp} \cap(\operatorname{Ker} C)^{\perp} \subseteq\left(\mathcal{W}_{g}\left(\mathcal{L}_{2}\right)\right)^{\perp} \\
& \left(\mathcal{W}_{g}^{*}\left(\mathcal{L}_{2}\right)\right)^{\perp} \cap(\operatorname{Ker} C)^{\perp} \subseteq\left(\mathcal{W}_{g}\left(\mathcal{L}_{1}\right)\right)^{\perp}
\end{aligned}
$$

Since conditions of Theorem 4.1 are satisfied, faults are isolable and using Theorem 3.1 (see 
(M2) section 4) too.

To conclude the filter form is proposed:

$$
\left\{\begin{array}{l}
\dot{z}_{1}=-z_{1}+u_{1} \\
\dot{z}_{2}=z_{1}-z_{2} \\
\dot{z}_{3}=z_{1}-z_{3}+u_{2} \\
\dot{z}_{4}=z_{2}-z_{4} \\
r_{1}=y_{4}-z_{4}
\end{array}\right.
$$

where residual $r_{1} \longrightarrow 0, \forall\left(u, m_{2}\right)$ and $r_{1} \neq$ 0 if $m_{1} \neq 0$ whereas,

$$
\left\{\begin{array}{l}
\dot{z}_{1}=-z_{1}+u_{1} \\
\dot{z}_{2}=z_{1}-z_{2} \\
\dot{z}_{3}=-z_{3}+u_{2}+y_{2}^{(2)}+2 y_{2}^{(1)}+y_{2} \\
\dot{z}_{4}=z_{2}-z_{4} \\
r_{2}=y_{1}-z_{3}
\end{array}\right.
$$

where residual $r_{2} \longrightarrow 0, \forall\left(u, m_{1}\right)$ and $r_{2} \neq$ 0 if $m_{2} \neq 0$.

\section{CONCLUSION}

In this paper, the problem of fault residual generation is considered. In one hand, equivalent Massoumnia's results are explained. In other hand, based on a new filter form with better fault decoupling results, conditions for fault isolation are proposed. Moreover it is shown that these conditions are no more restrictive. Finally, an example highlights these results. To answer applicability questions, the reader can refer to the articles (Fliess and Sira-Ramirez, 2003; Fliess and Sira-Ramirez, 2004) where some techniques stemming from differential algebra allow to obtain time derivatives of measured signals. In this work, the convergence analysis is not considered but is the future prospects. Results already obtained in (Lohmiller and Slotine, 1998; Join et al., 2002) are promising.

\section{REFERENCES}

Beard, R. V. (1971). Failure accomodation in linear systems through self-reorganization. PhD thesis. Massachusette Institute of Technologiy. Departement of Aeronautics and Astronautics. Cambridge, Massachusetts (USA).

Edelmayer, A., J. Bokor and F. Szigeti (1999). Detection filter design in uncertain linear systems: Application of the generalized observer scheme. In: $14^{\text {th }}$ IFAC World Congress on Automatic Control. Beijing, China.

Fliess, M. and H. Sira-Ramirez (2003). An algebraic framework for linear identification. ESAIM: Control, Optimisation an Calculus of Variations 9, 151-168.
Fliess, M. and H. Sira-Ramirez (2004). Control via state estimations of some nonlinear systems. In: Symp. Nonlinear Control Systems (NOL$C O S)$. Stuttgart, Allemagne.

Fossard, A. J. and D. Normand-Cyrot (1995). Nonlinear systems. Vol. 1-3. Chapman \& Hall. London, New York.

Frank, P. M. and X. Ding (1997). Survey of robust residual generation and evaluation methods in observer-based fault detection systems. Journal of Process Control 7(6), 403-424.

Glumineau, A., C. H. Moog and F. Plestan (1996). New algebro-geometric conditions for linearization by inpu-output injection. IEEE Transaction on Automatic Control 41(4), 598-603.

Isidori, A., A. J. Krener, C. Gori-Giorgi and S. Monaco (1981). A geometric approach to synthesis of failure detection filters. IEEE Transaction on Automatic Control 26(2), 331345.

Join, C., J. Jouffroy, J.-C. Ponsart and J. Lottin (2002). Synthse d'un filtre isolateur de dfauts l'aide de la thorie de la contraction. In: Confrence Internationale Francophone d'Automatique. Nantes, France. pp. 153-158.

Jones, H. L. (1973). Failure detection in linear systems. PhD thesis. Massachusette Institute of Technologiy. Departement of Aeronautics and Astronautics. Cambridge, Massachusetts (USA).

Kailath, T. (1980). Linear systems. Prentice Hall information and system science series. Prentice Hall. Englewood Cliffs.

Lohmiller, W. and J.J. E. Slotine (1998). Fault diagnosis in dynamic systems using analytical and knowledge-based redundancy a survey and some new results. Automatica 34(6), $683-696$.

Massoumnia, M.-A. (1986). A geometric approach to synthesis of failure detection filters. IEEE Transaction on Automatic Control 31(9), $839-846$.

Massoumnia, M.-A, G. C. Verghese and A. S. Willsky (1989). Failure detection and identification. IEEE Transaction on Automatic Control 34(3), 316-321.

Park, J., G. Rizzoni and W. B. Ribbens (1994). On the representation of sensor faults in fault detection filters. Automatica 30(11), $1793-$ 1795.

Plestan, F. and A. Glumineau (1996). Linearization by generalized input-output injection and synthesis of observers. In: IFAC 13th Triennial World Congress. San Francisco. pp. 281-285.

Wonham, W. M. (1985). Linear multivariable control: a geometric approach. volume 10 of Applications of Mathematics. $2^{\text {th }}$ ed.. SpringerVerlag. New York, Berlin, Heidelberg. 